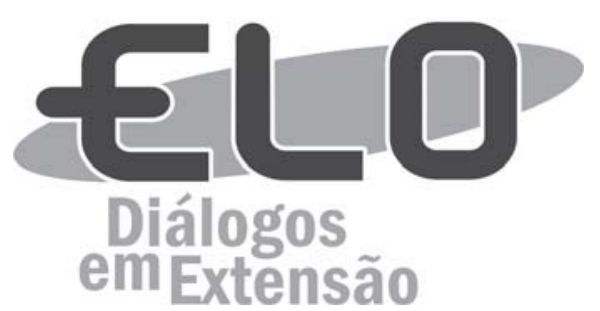

\title{
Origem e histórico da "Rede Nós de Água": pesquisa, ensino e extensão participativa em conservação de recursos hídricos sob a perspectiva agroecológica
}

\author{
Luan Ritchelle Aparecido dos Anjos ${ }^{1}$; Thais de Carvalho Maia ${ }^{2}$; Pedro \\ Henrique da Silva Queiroz ${ }^{3}$; Felipe Salgado de Senna ; Raquel Amorim \\ Campos ; Tommy Flávio Cardoso e Wanick Loureiro de Sousa ${ }^{6}$.
}

\begin{abstract}
Resumo: A "Rede Nós de Água" se origina de um processo de construção e sistematização de experiências agroecológicas emergentes na região de Araponga/MG no início dos anos 90, a partir de uma parceria entre o Centro de Tecnologias Alternativas da Zona da Mata (CTA-ZM), a Universidade Federal de Viçosa (UFV) $e$ agricultores familiares da região. Dessas experiências, surge a demanda por um melhor entendimento sobre a dinâmica dos recursos hídricos nesses sistemas agroflorestais, incitando a criação, em 2009, do Grupo de Trabalho das Águas (GT-Água). A partir dai, inúmeras ações de formação e pesquisa foram iniciadas e outras entidades se associaram ao grupo, constituindo, em 2014, a Rede Nós de Água, que atua em diversos municípios da Zona da Mata mineira e além, com técnicas e tecnologias sociais que buscam promover a conservação de recursos hídricos por meio de metodologias colaborativas e participativas, visando a autonomia e construção coletiva do conhecimento agroecológico.
\end{abstract}

Palavras-chave: Agroecologia. Educação ambiental. Plantio de água. Saneamento rural. Tecnologia social.

Área Temática: Agroecologia e Educação.

Origin and history of the "Rede Nós de Água": research, teaching and participatory extension in the conservation of water resources through the agroecological perspective

\begin{abstract}
The "Rede Nós de Água" originates from a process of construction and systematization of emerging agroecological experiences in Araponga/MG region in the early 1990s, based on a partnership between the Alternative Technologies Center of Forest Zone (CTA-ZM), the Federal University of Viçosa (UFV) and family farmers from the region. From these experiences, a demand for a better understanding of the dynamics of water resources in these agroforestry systems arises, prompting the creation of the Working Group on Water (GT-Água), in 2009. Since then, numerous training and research actions have been initiated and other entities have joined the group, constituting, in 2014, the Water Nodes Network, which operates in several cities in the Forest Zone of Minas Gerais and beyond, using social technologies and techniques which promote the conservation of water resources through collaborative and participatory methodologies, aiming the autonomy and collective construction of agroecological knowledge.
\end{abstract}

Keywords: Agroecology. Environmental education. Rural sanitation. Social technology. Water planting.

\footnotetext{
${ }^{1}$ Universidade Federal de Viçosa (UFV), Rua Álvaro Gouveia, nº 175, apto 202, centro, Viçosa/MG, Tel: (31) 983 413 114, luanraanjos@gmail.com.

${ }^{2}$ Universidade Federal de Viçosa (UFV).

${ }^{3}$ Universidade Federal de Viçosa (UFV).

${ }^{4}$ Instituto Socioambiental de Viçosa (ISA-Viçosa)

${ }^{5}$ Universidade Federal de Viçosa (UFV).

${ }^{6}$ Universidade Federal de Viçosa (UFV).
} 


\section{Origen e histórico de la "Rede Nós de Água": investigación, enseñanza y extensión participativa en la conservación de los recursos hidricos bajo perspectiva agroecológica}

Resumen: La "Rede Nós de Água" se origina de un proceso de construcción y sistematización de experiencias agroecológicas emergentes en la región de Araponga / MG a principios de los años 90, a partir de una asociación entre el Centro de Tecnologías Alternativas de la Zona de la Mata (CTA- ZM), la Universidad Federal de Viçosa (UFV) y los agricultores familiares de la región. De estas experiencias, surge la demanda por el mejor entendimiento sobre la dinámica de los recursos hídricos en estos sistemas agroforestales, incitando a la creación, en 2009, del Grupo de Trabajo de las Aguas (GT-Agua). A partir de ahi, innumerables acciones de formación e investigación se iniciaron y otras entidades se fueron asociando al grupo, constituyendo, en 2014, la Rede Nós de Água, que actúa en diversos municipios de la Zona de la Mata minera y además, con técnicas y tecnologías sociales que promuevan la conservación de los recursos hídricos a través de metodologías colaborativas y participativas, visando la autonomía y construcción colectiva del conocimiento agroecológico.

Palabras clave: Agroecología. Educación ambiental. Plantación de agua. Saneamiento rural. Tecnología social.

\section{Introdução}

A expansão dos sistemas de produção agropecuários e o crescimento da população mundial têm aumentado significativamente a demanda por água potável regularmente disponível no planeta. Em contrapartida, nos últimos anos têm-se verificado redução na precipitação e na distribuição das chuvas ao longo do período das águas, causando sérias preocupações quanto ao panorama do abastecimento de água (Marengo, 2008).

Apesar de a demanda hídrica ter aumentado significativamente, uma das principais causas da escassez desse recurso é a baixa eficiência relacionada à captação da água das chuvas pelo solo. Essa baixa eficiência tem provocado menor acúmulo de água nos lençóis freáticos e maior escoamento superficial, na forma de enxurradas, causando perda de solos agrícolas com a erosão e transtornos urbanos, como enchentes. Segundo Diagnóstico Rápido Participativo (DRP) realizado por Cardoso et al. (2001), a erosão foi a principal causa do decréscimo da capacidade produtiva dos solos na região de Araponga/MG. Diante disso, algumas ações foram iniciadas pelos agricultores locais em parceria com ONGs, professores e estudantes da Universidade Federal de Viçosa na busca de soluções para o problema.

A Rede Nós de Água, uma dessas iniciativas, está vinculada a projetos de educação ambiental por meio de cursos de capacitação, debates nas zonas rurais e oficinas socioambientais em Escolas Família Agrícola da Zona da Mata, além de práticas de plantio de água e saneamento rural. Essas atividades têm sido desenvolvidas nos municípios de Sem Peixe, Divino, Araponga, Jequerí, Cajurí, Paula Cândido, Catas Altas, Viçosa, e têm se estendido para outros municípios do estado.

\section{Objetivos}

Objetiva-se, com este artigo, descrever a origem e o histórico da Rede Nós de Água, ressaltando o contexto socioambiental do trabalho desenvolvido e os diferentes aspectos da pesquisa, ensino e extensão participativa em conservação de recursos hídricos sob a perspectiva agroecológica trabalhados pela rede.

\section{Metodologia}

No início da década de 1990, uma parceria entre o Centro de Tecnologias Alternativas da Zona da Mata (CTA-ZM), a Universidade Federal de Viçosa (UFV) e agricultores familiares da região de Araponga/MG deu inicio a uma série de experimentações com Sistemas Agroflorestais - modelos de cultivo utilizados como alternativa às práticas convencionais de cultivo do café na região. Os estudos desses sistemas de produção, baseados na diversidade de plantas, buscavam, principalmente, melhorias na capacidade produtiva das culturas e na qualidade dos solos. Os resultados obtidos indicaram melhoria da fertilidade dos solos, do aumento de biodiversidade e da quantidade de águas oriunda das propriedades. 
Há, basicamente, dois tipos de sistemas de produção empregados nessas áreas: o modelo agroecológico de cultivo, baseado na diversificação de culturas, no uso de insumos orgânicos, no cultivo sem uso de agrotóxicos e na cobertura do solo; e o sistema convencional, baseado na monocultura do café, com o uso de insumos químicos industrializados e agrotóxicos.

A partir daí, os sistemas agroflorestais foram avaliados sob o ponto de vista de sua contribuição para os recursos hídricos, além dos demais benefícios ao solo e à biodiversidade. Nesse contexto, surgiram demandas dos agricultores no sentido de se avaliar o impacto dos sistemas de produção do café praticados na região sobre a Bacia do Córrego São Joaquim.

A partir dessa e de outras demandas foi criado o Grupo de Trabalho das Águas (GT-Água) no ano de 2009, com o objetivo de avaliar o impacto dos sistemas de produção agrícola aos recursos hídricos e vislumbrar estratégias de mitigação que aliem a produção agrícola à conservação dos ecossistemas terrestre e aquático, avaliando qualitativamente a condição da água proveniente desses sistemas de produção e quantitativamente a sua eficiência de manutenção da regularidade da vazão nas nascentes existentes em cada sistema, por meio da maior ou menor exploração da capacidade de recarga dos lençóis freáticos.

Dessa forma, o grupo GT-Água veio promovendo um trabalho de pesquisa-ação nessas e em outras áreas da região com a participação e acompanhamento das comunidades envolvidas, permitindo descobrir as possíveis causas do deficit hídrico comumente observado, avaliar as consequências desse impacto a curto, médio e longo prazo e propor soluções para tal problema.

Em meados de 2007, surge o Instituto Socioambiental de Viçosa (ISA Viçosa), organização não governamental (ONG) com o objetivo de incentivar e disseminar práticas que visem a conservação da bacia do Ribeirão São Bartolomeu - principal fornecedor de água para o sistema de abastecimento da cidade de Viçosa/MG - e promover cursos de Educação Ambiental na região de Viçosa.

A partir de uma articulação conjunta do GT-Água, do ISA-Viçosa, do CTA e da Associação dos Plantadores de Água (Plantágua), de Alegre - ES, foi realizado um curso de Plantio de Água, em 2014, na cidade de Viçosa/MG, de onde surge a demanda de criar uma entidade comum que reúna todos os grupos envolvidos com a conservação de recursos hídricos na região. Criou-se, portanto, a Rede Nós de Água, que tem atuado diretamente em diversas áreas estratégicas de mais de 10 municípios da Zona da Mata Mineira.

Concomitantemente à criação da Rede Nós de Água, ocorria uma importante articulação que visava reunir todos os grupos ligados à agroecologia que atuam na Universidade Federal de Viçosa. Esses grupos são oriundos da universidade, mas se estendem por diversas regiões da Zona da Mata Mineira e se articulam com prefeituras, Sindicatos de Trabalhadores Rurais, associações e cooperativas de agricultores e Escolas Família Agrícola de toda a região. O núcleo de Educação do Campo e Agroecologia (ECOA), institucionalizado em 2016, é um importante espaço de articulação das organizações sociais e sujeitos que compõem a rede do movimento agroecológico e da educação do campo da Zona da Mata mineira. O núcleo conecta e aproxima as iniciativas em curso, de modo a potencializar a construção coletiva de ações em prol da educação do campo e da agroecologia, por meio do desenvolvimento de programas e projetos de ensino, pesquisa e extensão dentro da dinâmica do tecido social existente (Da Mata Silveira, 2017).

O ECOA, juntamente com o curso de Licenciatura em Educação do Campo e a Escola de Energia Popular de Viçosa (ENEP) compõem e alimentam a rede Nós de Água.

A Rede Nós de Água foi inspirada na experiência do agricultor familiar Newton Campos, morador de uma comunidade rural de Alegre, município do estado do Espírito Santo. Segundo Meira et al. (2013), durante a década de 1980, esse agricultor herdou uma pequena propriedade que fora desmatada e utilizada para criação de animais, sendo a maior parte da área ocupada por pastagens degradadas e poucos recursos hídricos disponíveis. Na tentativa de reverter a situação da sua propriedade, ele adotou uma série de medidas conservacionistas, como cercamento de nascentes, reflorestamento, captação da água das chuvas por caixas secas, recuperação das matas ciliares e topos de morros com auxílio de grupos de extensão universitária, pesquisa e outras instituições. As ações geraram resultados expressivos na recuperação da área degradada, sobretudo quanto à quantidade de água oriunda da propriedade, e o senhor Newton passou a receber crianças e adolescentes com intuito de transmitir seu conhecimento por meio da educação ambiental. A esse conjunto de técnicas mitigadoras de baixo custo que foram adotadas visando a conservação dos recursos hídricos por meio aumento de infiltração da água da chuva no solo deu-se o nome de "Plantio de Água". 
Portanto, a Rede Nós de Água visa disseminar técnicas e tecnologias sociais para o "Plantio de Água", buscando a construção do conhecimento agroecológico e a formação de "plantadores de Água", visando a preservação qualitativa e quantitativa dos recursos hídricos.

Com o objetivo de aumentar a eficiência de captação hídrica e melhorar a qualidade da água e dos solos, a rede vem atuando fortemente na formação de uma identidade de "Plantio de Água" nas comunidades rurais, visando formar multiplicadores e disseminadores de técnicas e tecnologias sociais para o aumento da quantidade e melhoria na qualidade da água proveniente das nascentes das propriedades. Esse trabalho é realizado por intermédio de cursos e mutirões coletivos para a construção das estruturas de captação de água, que melhoram a eficiência da infiltração da água nos solos. As técnicas e tecnologias sociais utilizadas para o aumento na quantidade de água são: o cercamento e proteção de nascentes, construção de caixas secas e cheias, e o terraceamento em curva de nível. Para a melhora na qualidade da água devolvida ao meio ambiente são construídas fossas sépticas biodigestoras e evapotranspiradoras. Todas as ações são realizadas por meio de metodologias participativas e colaborativas, propondo a autonomia e construção coletiva do conhecimento agroecológico.

Para difusão e apropriação dos métodos, nas propriedades onde foram implementadas as técnicas, são realizados intercâmbios agroecológicos, como descrito por Zanelli et al., 2015, com o objetivo de incluir novos atores nas ações da rede e aumentar a difusão do conhecimento agroecológico em relação às técnicas de plantio de água.

Entendendo a necessidade de capacitar os jovens do meio rural, já que os mesmos serão potenciais agricultores e agricultoras, a rede atua em projetos de educação ambiental nas zonas rurais da Zona da Mata mineira. Atualmente existe um projeto de educação ambiental na Escola Família Agrícola Puris de Araponga/MG (EFA-Puris), realizado com estudantes do ensino médio desde 2015 com temas que vão desde a conservação de recursos hídricos e solo à construção de estruturas para o plantio de água e saneamento rural.

\section{Resultados e Discussão}

As atividades da rede abrangem a dimensão de extensão, com destaque para os cursos e atividades de Plantio de Água (Figura 1), construção de fossas sépticas ecológicas (evapotranspiradoras e biodigestoras) (Figura 2) e cursos de saneamento rural, atividades de Educação Ambiental nas Escolas Família Agrícola da região (Figura 3) e demais atividades de formação de agricultores, por meio de oficinas e cursos. Com relação à pesquisa, a rede tem estudado a relação dos Sistemas Agroflorestais (SAFs) de produção agrícola e os recursos hídricos produzidos nas propriedades rurais, quantitativa e qualitativamente; além da eficiência dos métodos de saneamento rural, representado pelas fossas ecológicas e o impacto toxicológico do uso de pesticidas nas bacias hidrográficas. Nesse contexto, sob financiamento da Fapemig, CNPq, Fundação Banco do Brasil, CAPES, SEDA e convênios com a prefeitura de Viçosa e Secretaria de Estado, a rede já participou de duas dissertações de mestrado, diversos trabalhos de conclusão de curso (TCC), diversos projetos de extensão com interface em pesquisa pelo Programa Institucional de Bolsas de Extensão Universitária (PIBEX), e dezenas de resumos em simpósios e congressos.

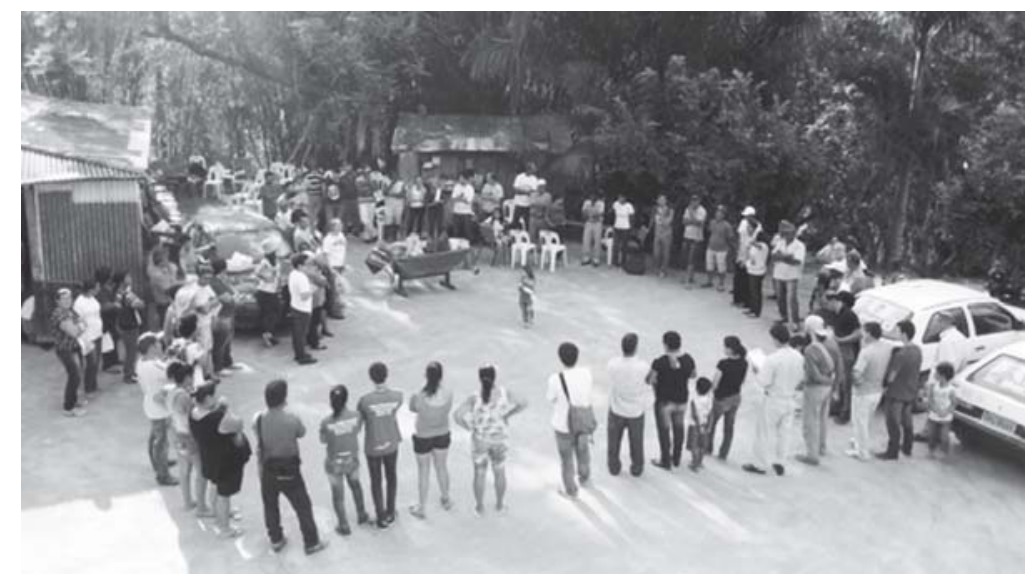

Figura 1 - Curso de Plantio de Água para agricultores familiares do município de Divino/MG. Fonte: acervo Rede Nós de Água. 


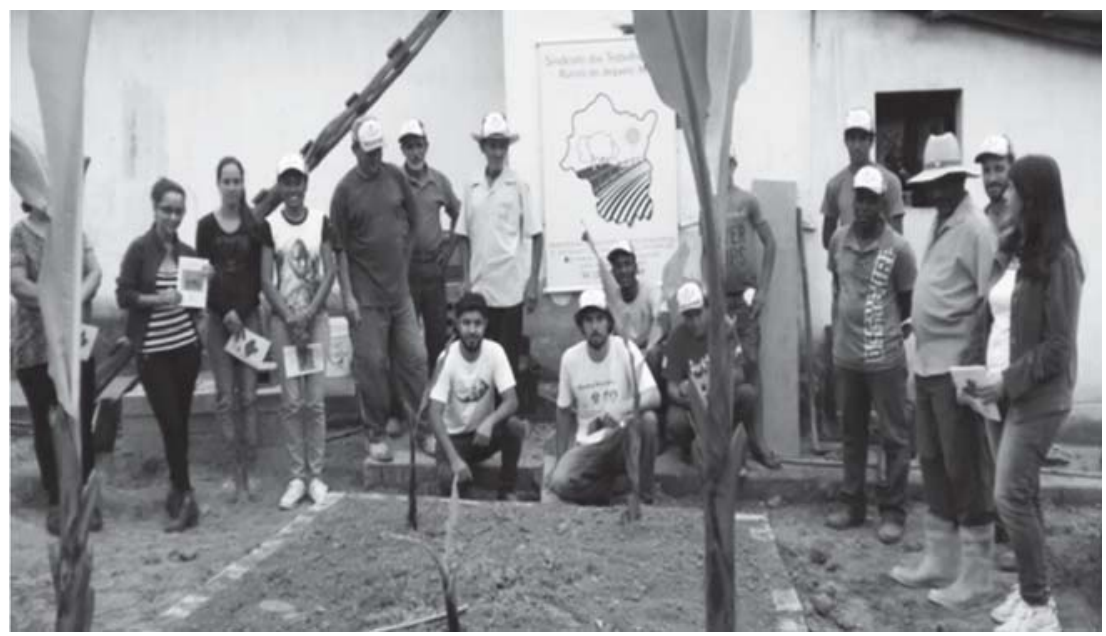

Figura 2 - Fossa evapotranspiradora coletivamente construída por agricultores familiares durante um mutirão de formação.

Fonte: acervo Rede Nós de Água.

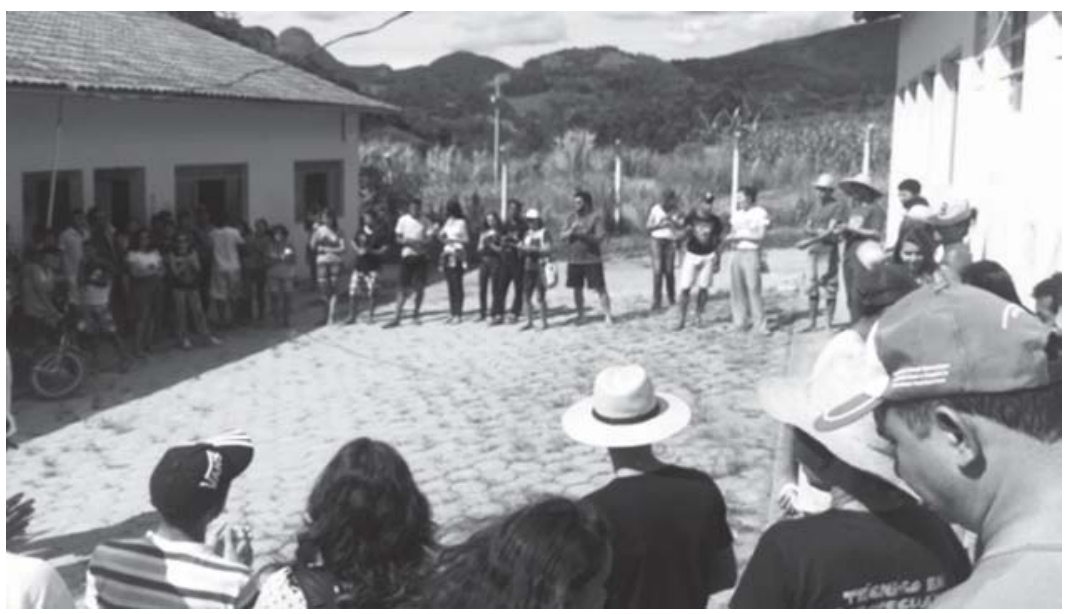

Figura 3 - Aula prática para formação de estudantes da Escola Família Agrícola Puris de Araponga/ MG (EFA-Puris) em Educação Ambiental.

Fonte: acervo Rede Nós de Água.

A difusão de técnicas e tecnologias sociais nos processos de formação idealizados pela rede possuem um enfoque metodológico participativo e adaptado às diversas realidades. Além disso, esse processo tem promovido a formação do sujeitos envolvidos, tornando-os aptos a aplicar, difundir e disseminar todo o conhecimento teórico e prático trabalhado, possibilitando uma transmissão horizontal do conhecimento. A presença de um grande número de estudantes de EFA's e do curso de Licenciatura em Educação do Campo (LICENA/UFV) nos processos de formação permite que as técnicas e tecnologias alcancem todo o estado de Minas Gerais e diversas regiões de outros estados.

\section{Conclusões}

Portanto, desde 2009, diversas ações têm sido realizadas visando a sensibilização para uma consciência ambiental e agroecológica, associada à difusão de tecnologias sociais para a conservação e manutenção de nascentes e de cursos d'água nas propriedades rurais, buscando garantir a autonomia das famílias e a sustentabilidade dos recursos naturais.

Os trabalhos desenvolvidos pela rede têm contribuído para a construção de um pensamento crítico e consciente em relação a água por parte da população, servindo de subsídio para a realização de serviços ambientais, saneamento rural e de recuperação e conservação de recursos hídricos. E, a partir disso, estão sendo realizadas atividades pra a construção de soluções para os problemas traçados pelos próprios agricultores. 


\section{Agradecimentos}

Aos agricultores (as) locais de Araponga, Viçosa, Divino, Sem Peixe e demais zonas rurais que fazem parte da história da rede, aos Sindicatos de Trabalhadores Rurais, Escola Família Agrícola, CTA - Zona da Mata, UFV, aos órgãos de financiamento Fapemig, CNPq, Fundação Banco do Brasil, CAPES, SEDA e convênios com a prefeitura de Viçosa e Secretaria de Estado.

\section{Referências bibliográficas}

CARDOSO, Irene M. et al. Continual learning for agroforestry system design: university, NGO and farmer partnership in Minas Gerais, Brazil. Agricultural Systems, v. 69, n. 3, p. 235-257, 2001.

DA MATA SILVEIRA, Maysa et al. Núcleo de educação do campo e agroecologia (ECOA): uma construção plural e coletiva. Cadernos de Agroecologia, v. 12, n. 1, 2017.

MARENGO, José Antônio. Água e mudanças climáticas. Estudos avançados, v. 22, n. 63, p. 83-96, 2008.

MEIRA, Ana Cláudia Hebling; et al. Capacitação de Multiplicadores para plantio de água. 1 ed. Alegre: Projeto Plantadores de Água. 2013.

ZANELLI, Fabrício V. et al. Intercâmbios agroecológicos: aprendizado coletivo. Informe Agropecuário, Belo Horizonte, v. 36, n. 287, p. 9-23, 2015.

Recebido para publicação em 30/10/2017 e aprovado em 15/05/2018. 